\title{
Étude et modélisation du front d'onde des nouvelles sources XUV
}

\author{
S. Le Pape, Ph. Zeitoun, J.-F. Hergott ${ }^{1}$, B. Carré ${ }^{1}$, P. Dhez, M. François ${ }^{2}$, M. Idir, \\ H. Merdji ${ }^{1}$, D. Ros, A. Carillon et P. Salières ${ }^{1}$ \\ Laboratoire de Spectroscopie Atomique et lonique, bâtiment 350, Université Paris Sud, \\ 91405 Orsay, France \\ ${ }^{1}$ CEA SPAM/DSM, Centre d'Étude de Saclay, 91191 Gif-sur-Yvette, France \\ 2 Institut d'Electronique et de Microélectronique du Nord, Université des Sciences \\ et Technologie de Lille, avenue Poincaré, BP. 69, 59652 Villeneuve-d'Ascq, France
}

\begin{abstract}
Résumé :Un nouveau champ d'expériences dans le domaine XUV serait ouvert si une intensité de typiquement $10^{13} \mathrm{~W} / \mathrm{cm}^{2}$ etait atteinte. Pour atteindre cette intensité, il faut focaliser le rayonnement XUV sur des taches focales de dimension micronique, ce qui nécessite une source dont le front d'onde est très régulier. Nous nous sommes donc intéressé aux problèmes liés à la focalisation des sources nouvelles sources XUV (laser X et harmoniques d'ordre élevé) et en particulier à leur front d'onde. Nous avons développé le premier senseur de front d'onde de type "Shack-Hartmann " fonctionnant dans l'XUV. Nous montrons ici les premiers résultats obtenus avec ce senseur de front d'onde ainsi que les simulations développées en parallèle.
\end{abstract}

\section{INTRODUCTION}

Les nouvelles sources XUV, la génération d'harmoniques d'ordre élevé (HOE), les lasers X pompés par laser ou par décharge électrique, sont désormais utilisés pour des expériences d'applications ${ }^{1}$. Des expériences d'interférométrie pour sonder la densité électronique d'un plasmal, ou pour visualiser les défauts à la surface d'un supraconducteur sous champ électrique intense ont été réalisés avec succès ${ }^{2}$. A notre connaissance, il n'a pas encore été observé de phénomènes non-linéaires dans l'XUV $(\lambda<40 \mathrm{~nm})$ qui ne peuvent apparaître qu'à des intensités de l'ordre de $10^{12}-10^{13} \mathrm{~W} / \mathrm{cm}^{2}$. Pour atteindre cette intensité, des expériences de focalisation de laser XUV ont été réalisées ${ }^{3}$ à l'ILE au Japon et au LULI en France. La dimension estimée des taches focales obtenues lors de ces expériences a semblé très grande par rapport à l'optimum attendu ce qui laissait supposer que le front d'onde était peu régulier. Suite à ces expériences préliminaires, nous avons commencé une étude générale des problèmes liés à la focalisation des nouvelles sources XUV. Ce travail consiste en une étude de la qualité de focalisation de l'optique utilisée (lentille de Bragg-Fresnel), suivie d'une étude expérimentale et numérique du front d'onde de la source.

\section{ETUDE DE LA FOCALISATION DE L'HARMONIQUE $37(\lambda=21.6 \mathrm{~nm})$ D'UN LASER Ti:SAPHIR}

Dans le cas de sources ayant un fort taux de répétition, comme la génération d'harmoniques d'ordre élevée $(10 \mathrm{~Hz})$, on utilise une technique dite de foucaultage pour étudier la qualité de focalisation de la source. Le principe de la mesure consiste à déplacer, dans un plan perpendiculaire à la propagation, une fente placée au foyer de la lentille et à enregistrer le signal transmis. Le plus souvent on dérive cette courbe pour obtenir la répartition d'intensité. Nous avons utilisé cette technique lors d'une expérience réalisée au CEA-SPAM à Saclay pour étudier la focalisation d'une harmonique d'ordre élevé (37 ${ }^{\text {màe }}$ $\lambda=21.6 \mathrm{~nm}$ ) générée dans du néon.

L'optique focalisante utilisée lors de cette expérience était une lentille de Fresnel en réflexion (BraggFresnel). Une lentille de Fresnel est une lentille diffractive, le réseau est constitué d'ellipses 
concentriques. Cette lentille a été réalisée en 3 étapes : écriture du motif sur une résine par un faisceau d'électrons, développement du motif par attaque chimique, dépôt d'un traitement multicouche interférentiel réflecteur. Le dernier trait a une largeur de $0.14 \mu \mathrm{m}$. La figure 1 montre la tache focale optimale mesurée lors de cette étude.

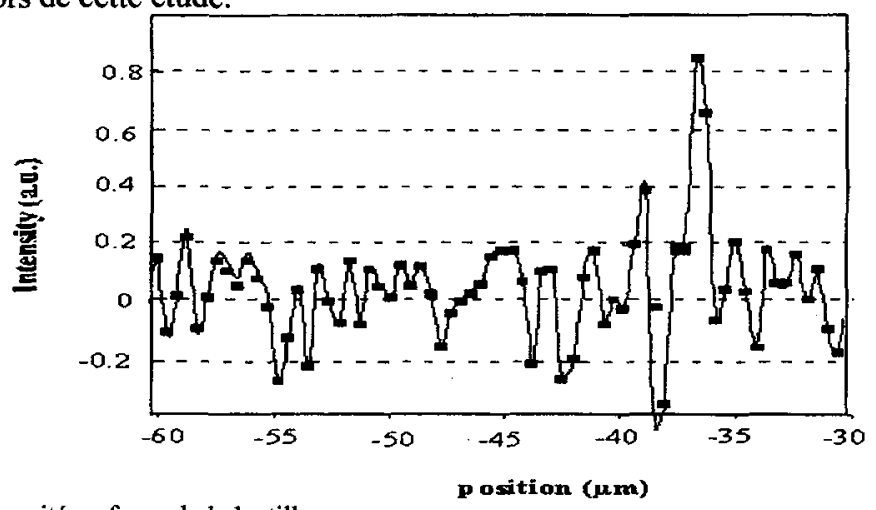

Fig.1 : Répartition d'intensité au foyer de la lentille

La dimension de cette tache focale (pic à $-36 \mu \mathrm{m}$ sur la figure 1) est de $1 \times 2.3 \mu \mathrm{m}^{2}$, on estime alors un l'éclairement de l'ordre de $10^{10} \mathrm{Wcm}^{-2}$ à $58 \mathrm{eV}$. Cette valeur pourrait être nettement augmentée en utilisant les nouvelles techniques de génération HOE qui semblent montrer une augmentation du nombre de photons émis d'au moins un ordre de grandeur, ou alors en travaillant vers $40 \mathrm{~nm}$, ce qui permet d'utiliser le xénon qui produit environ 100 fois plus de photons. La figure 1 est aussi intéressante à un autre point de vue. On observe de nombreuses structures autour de la tache focale principale, qui semblent être des pics de diffraction ou d'interférences. Durant des expériences ultérieures de telles structures ont été clairement observées en plaçant une caméra CCD (Charge Coupled Device) au foyer des lentilles. Or, les modèles classiques ne font apparaître aucune figure de diffraction ou d'interférence. Il semble que ce désaccord expérience-théorie provient de l'absence de prise en compte de la cohérence transverse de la source qui, dans le cas des harmoniques d'ordre élevé, est très forte. On observe aussi une légère asymétrie de la tache focale principale. Celle-ci a deux origines. D'une part, le creux vers une position de $-37 \mu \mathrm{m}$ est dû à la variation tir à tir de l'intensité du faisceau harmonique, amplifié par la dérivation du signal brut. D'autre part, en dehors de ce creux, on peut considérer que l'asymétrie provient partiellement d'un défaut résiduel soit sur le front d'onde du faisceau harmonique, soit sur le réglage de la lentille. Ce genre de résultat illustre l'importance de dissocier les mesures de front d'onde de la source, des tests de qualités des optiques. La suite de ce texte présente les premières expériences de mesures de front d'onde d'une source XUV.

\section{CARTOGRAPHIE DES VECTEURS D'ONDE D'UN LASER X}

\subsection{Expérience de Shack-Hartmann}

Le principe du Shack-Hartmann (SH) est d'échantillonner un faisceau en décomposant la pupille en souspupilles. Ceci est réalisé par une matrice de lentilles. Chaque sous-lentille focalise alors la lumière sur un détecteur placé au foyer. Pour remonter au front d'onde, on compare la position de chaque tache à celle obtenue avec une onde dite de référence, qui est parfaitement connue. Cette onde est le plus souvent obtenue en faisant diffracté un faisceau à la même longueur d'onde sur un microtrou $(0.1$ à $1 \mu \mathrm{m})$, générant ainsi une onde sphérique. Cette technique permet aussi d'éviter de mesurer la déformation du front d'onde induite par le système d'analyse. En utilisant un e onde de référence, nous obtenons ainsi.le front d'onde en absolu. Précisons, que dans le cas où la source étudiée est spatialement cohérente sur 
toute la dimension du senseur de front d'onde, on peut alors reconstruire la surface d'onde de la source étudiée (cas des HOE), tandis que dans le cas d'une source partiellement cohérente (laser X) on obtient la cartographie de l'inclinaison des vecteurs d'onde. Ces deux grandeurs contiennent en final les mêmes informations sur la qualité du faisceau.

Malgré l'utilisation d'une onde de référence, nous avons choisi de travailler en réflexion, assurant ainsi une bonne co-planéité des lentilles. La matrice comporte $12 \times 10$ lentilles de Fresnel de $50 \mathrm{~cm}$ de longueur focale. La taille du dernier trait est de $13 \mu \mathrm{m}$. La distance entre deux centres de lentille adjacente est de $900 \mu \mathrm{m}$. La distance focale a été calculée de telle sorte qu'une inclinaison de $0,1 \mathrm{mrad}$ du vecteur d'onde, $\mathbf{k}$, soit observable ( 3 pixels de décalage sur le détecteur)

Dans le cas du laser X, l'inclinaison des vecteurs d'onde dépend beaucoup de la réfraction subie lors de la propagation du rayonnement dans le milieu amplificateur. La réfraction dans le plasma peut être atténuée en irradiant la cible par deux impulsions laser. La première impulsion, très faible par rapport à la seconde, a pour but de créer un pré-plasma de manière à « préparer » la cible avant l'arrivée de la seconde impulsion, et ainsi de réduire notablement la réfraction. Cette technique est utilisée depuis longtemps pour faire convenablement fonctionner le laser $\mathrm{X}$ du LULI/LSAI. Il y a quelques années, une étude poussée de l'effet de cette pré-impulsion sur le niveau d'émission du laser $X$ a été réalisée. Nous avons ainsi trouvé un niveau optimal pour la pré-impulsion. Cependant, quand on s'intéresse à focaliser le laser $\mathrm{X}$, il devient nécessaire de trouver un régime de fonctionnement pour lequel l'ouverture angulaire des vecteurs d'onde est la plus faible possible. Le senseur de front d'onde de type Shack-Hartmann est totalement adapté à cette étude. Les résultats sont encore préliminaires, mais permettent déjà de tirer certaines conclusions. Malheureusement, à cette époque nous ne disposions pas d'onde de référence. Les données ne peuvent être traitées qu'en comparant directement deux mesures.

En dépit de l'utilisation d'un détecteur nettement moins résolvant que prévue initialement (perte d'un facteur 10 sur la résolution), nous pouvons observer l'évolution de l'inclinaison des vecteurs d'onde en comparant directement deux images prises dans des conditions différentes. L'image de gauche de la figure 2 est la soustraction de deux images prises pour des niveaux de pré-impulsion de $5,3 \times 10^{-3}$ et de $5,8 \times 10^{-3}$ (rapport entre l'intensité de la pré-impulsion et de l'impulsion principale). Le laser $\mathrm{X}$ était ici le « classique " laser à $21.2 \mathrm{~nm}$ fonctionnant en double passage à l'aide d'une demi-cavité.
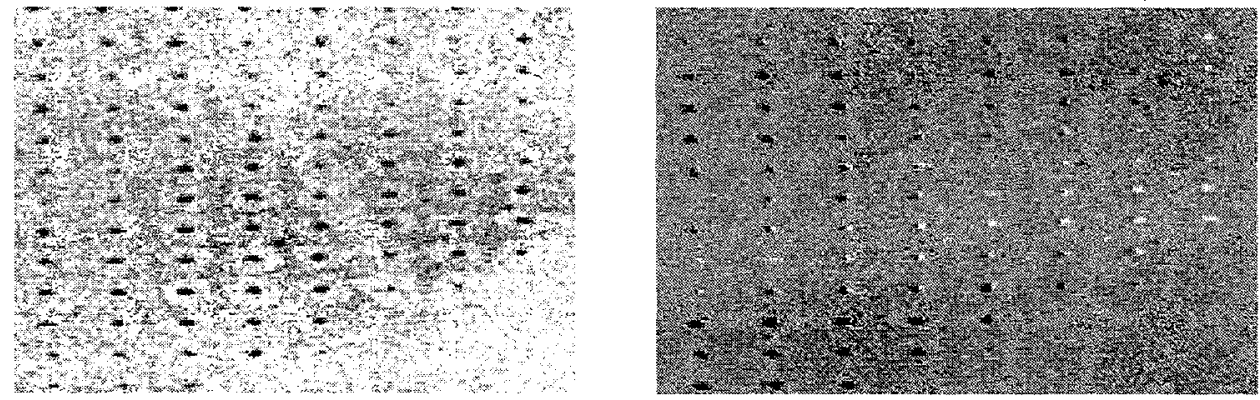

Fig 2 :: A gauche, on peut voir une image brute de SH. Les points noirs sont les taches focales. A droite est présentée la soustraction de deux images pour deux niveaux de pré-impulsion différents Des déplacements sont mis en évidence quand les taches présentent un mélange de couleur (noir+blanc).

On peut remarquer sur cette image un déplacement non-uniforme des points focaux. Le déplacement des taches focales n'est pas dû à un changement de pointé du laser X qui aurait engendré un déplacement uniforme. Il faut aussi remarquer la sensibilité de ce senseur. En effet, une variation notable des angles des vecteurs $k$ est mesurée pour une variation de seulement $10 \%$ du niveau de pré-impulsion! Or quand on s'intéresse uniquement à l'énergie contenue dans le faisceau (niveau et répartition), une si petite variation du niveau de la pré-impulsion ne produit aucun effet mesurable. Ce résultat montre que l'on 
peut contrôler finement l'émission laser X et permettre ainsi de le fiabiliser pour des applications, ou d'apporter des renseignements importants pour une meilleure compréhension de cette source. Des études complémentaires sont prévues.

\subsection{Modélisation du laser X}

Pour mieux comprendre les résultats expérimentaux donnés par ce senseur de front d'onde nous avons développé un code de tracé de rayons à trois dimensions -3D- (SHADOX) simulant la l'amplification du laser $\mathrm{X}$ pompé par laser.

Nous avons choisi de développer un code de tracé de rayons 3D car il permet de comparer directement les données expérimentales du SH aux résultats de la simulation. Ce code modélise le laser X en simple et multi-passage. SHADOX est un post-processeur au code hydro-atomique EHYBRID ${ }^{3}$ qui calcule l'évolution spatio-temporelle des paramètres du plasma : émissivité, gain, densité électronique et largeur du plasma. Les résultats que nous présentons, fig. 3, montrent l'influence de la demi-cavité sur la distribution angulaire des vecteurs d'onde.

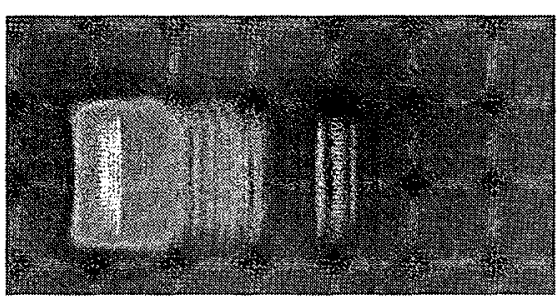

SIMPLE PASSAGE

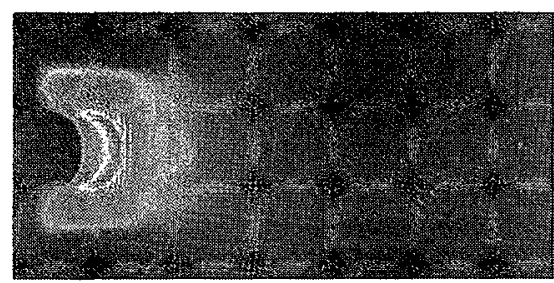

DOUBLE PASSAGE
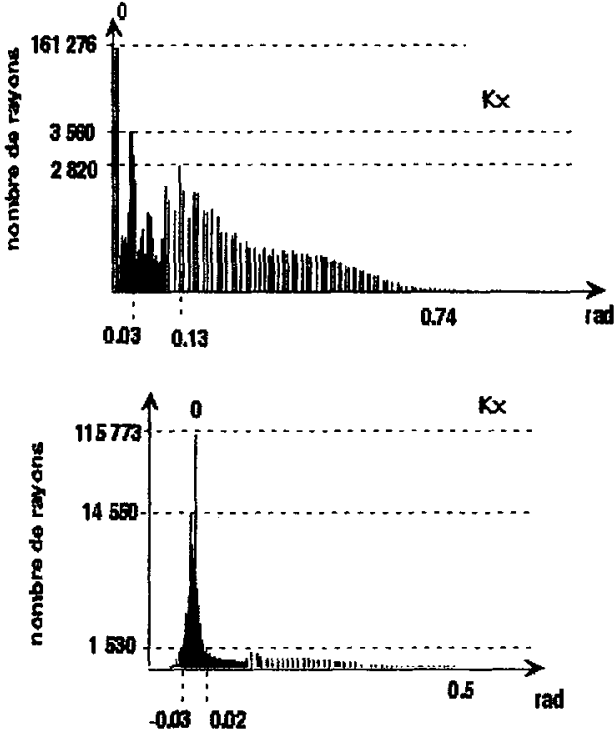

fig.3 :Distribution de la projection du vecteur d'onde dans le plan latéral (X). Données prises dans le plan de sortie du plasma. A droite sont présentés des histogrammes des dessins.

Les deux images de gauche présentent la distribution angulaire des vecteurs d'onde, en niveau de gris, dans la section du faisceau juste à la sortie du milieu amplificateur. En premier, on peut observer que le faisceau en simple passage est plus étendu que celui produit en double passage. Pour ce dernier, on remarque une courbure du faisceau liée à la réfraction subie par le laser $\mathrm{X}$ lors de l'amplification et donc à la structure des gradients de densité électronique dans le plasma. Pour mieux comprendre l'influence de la demi-cavité sur le faisceau laser $\mathrm{X}$, nous avons aussi présenté les histogrammes des figures. Ceux-ci donnent le nombre de rayons en fonction de l'angle des vecteurs d'onde projetés dans le plan horizontal. La comparaison des deux histogrammes montre une forte réduction du spectre angulaire quand le laser $\mathrm{X}$ est amplifié une deuxième fois à (double passage). L'ouverture angulaire des vecteurs d'onde passe de 0.2 mrad à $1 / 100 \mathrm{du}$ pic en simple passage, contre $0.05 \mathrm{mrad}$ en double. Ce résultat a deux conséquences. D'une part, le faisceau est beaucoup plus facile à focaliser, ce qui est évidemment un progrès. D'autre 
part, cette collimation des vecteurs d'onde doit se traduire aussi par une augmentation de la cohérence transverse (uniquement celle dite de propagation ${ }^{4}$ ) du laser $\mathrm{X}$. Cet effet, purement géométrique, a été observé expérimentalement au LULI pour les conditions considérées dans la modélisation.

\section{CONCLUSION}

Nous avons développé un senseur de front de type Shack-Hartmann susceptible de donner dans le cas d'une source partiellement incohérente une cartographie de l'inclinaison des vecteurs d'onde, et dans le cas d'une source spatialement cohérente sur toute la dimension du senseur de reconstruire la surface d'onde. Ce senseur a été testé pour la première fois sur un laser X. Les résultats, bien que préliminaires, montrent une influence importante du niveau de la pré-impulsion sur la répartition angulaire des vecteurs d'onde. Comparativement à des diagnostics basés uniquement sur l'énergie du faisceau laser X, la grande sensibilité de ce senseur en fait un élément important pour de futurs travaux d'optimisation du laser X. Nous avons aussi développé un code de tracé de rayons qui nous permet de comprendre les données expérimentales du Shack-Hartmann et d'envisager de nouvelles géométries du laser $\mathrm{X}$ (cavité complète). Nous avons ainsi montré que la réduction du spectre angulaire des vecteurs d'onde, observée expérimentalement, quand le laser $\mathrm{X}$ passe une deuxième fois dans le plasma est uniquement due à un effet géométrique lié à la structure réfractive du plasma. Nous avons aussi montré que notre code donne des informations sur la longueur de cohérence transverse.

\section{Référence}

1. L.B Da Silva, et al, Phys. Rev. Lett., 74 (20), 15 May 1995, p. 3991-3994

2. Ph . Zeitoun et al, Nucl. Inst. and Meth. in Phys. Res A, 416, p189 (1998)

3. G.J Pert, J. Fluid Mech.131 (1983) 401

4. M. Born and E. Wolf, « Principles of Optics », Pergamon Press, $6^{\text {th }}$ Edition, 1985 